\title{
Post-Cholecystectomy cystic bile Leaks: Why Endoscopically Overtreat is so Diffuse?
}

\author{
Massimiliano Mutignani, Edoardo Forti, Francesco Pugliese, Paola Fontana, Alberto Tringali, Marcello Cintolo \\ and Lorenzo Dioscoridi*
}

Digestive and Operative Endoscopy Unit, Niguarda-Ca' Granda Hospital, Italy

Submission: March 08, 2017; Published: June 16, 2017

"Corresponding author: Lorenzo Dioscoridi, Digestive and Operative Endoscopy Unit, Niguarda-Ca' Granda Hospital, Piazza dell'Ospedale Maggiore 3, 20121, Milan, Italy, phonenumber 00390264444883, Fax: 00390264442471; Email: dioscoridi.lorenzo@virgilio.it

\begin{abstract}
Candida albicans is the most common well known cause of infectious esophagitis. Although most patients with Candida esophagitis are immunocompromised, about $25 \%$ have scleroderma, achalasia, or other causes of esophageal dysmotility that would allow the fungi to overgrow and colonize the esophagus, with subsequent esophagitis $[1,2]$. Esophageal candidiasis usually manifests as white mucosal plaque-like lesions but it has been reported in the literature that it can manifest as an esophageal mass. We are reporting a case of recurrent esophageal candidiasis manifesting on the Esophagogastroduodenoscopy (EGD) as an obstructing mass.
\end{abstract}

\section{Mini Review}

Cholecystectomy is one of the most common surgical operation worldwide performed. Thus, postoperative complications are also common, including bleeding and bile leakages from the cystic stump [1]. Endoscopic treatment is usually performed for the management of the last type of complication $[1,2]$ moreover because the surgical re-intervention has a high percentage of morbidity and, if performed late, the cystic stump cannot be prepared so easily [1]. The endotheray required is just a biliary sphincterotomy to reduce the pressure gradient between the biliary tree and the duodenal lumen [3]. However, there are many papers present in the literature describing the association of biliary sphincterotomy and common bile duct's stenting (sometimes, with self-expandable metal stents) as treatment of choice for this type of complication. Hereafter, we report just few examples. The articles come from the research on PubMed-Medline inserting "CYSTIC DUCT LEAK+CHOLECYSTECTOMY+ENDOTHERAPY" as key words.

Rustagi et al. [4] describe the endoscopic stent placement during ERCP for the treatment of all type of biliary leaks after laparoscopic cholecystectomy. Adler et al. [5] show the results of a large series of patients treated endoscopically for traumatic or postsurgical bile duct leaks. Although the most common site of bile leak was the cystic duct remnant, they always describe an endoscopic stent placement [5]. David Martin et al. [6] write on bile duct injuries after laparoscopic cholecystectomy in a single- center series: a cystic stump dehiscence has been described in 4 cases, but in all the cases, a stent placement was performed. Ibrarullah et al. [7] describe the management of bile duct injury at various stages of presentation. In case of bile leakage but intact common bile duct, an endoscopic papillotomy with insertion of $10 \mathrm{Fr}$ plastic stent was always performed [7].

At this point, we would like to kindly remind to colleagues, first of all, the pathophysiological principles of this type of endotherapy (that are sometimes forgotten).

Normally, the intrabiliary pressure (mean value: $20 \mathrm{cmH} 20$ ) is major than intrabdominal pressure (mean value: $10 \mathrm{cmH} 20$ ). The presence of an abdominal drain modifies the intrabdominal pressure decreasing it till the atmospheric one (that is considered conventionally as 0 value) at the site of drain: bile comes out from bile duct into the abdominal drain if a leak is present. On the other hand, if a surgical drain was not placed at the time of surgery, the bile comes out from the dehiescence of the cystic stump and a bile collection is formed usually at the site of the previous cholecystectomy. Considering the pathophisiology of these types of bile leaks, we have to change the pressure gradient endoscopically in order to facilitate the bile inflow from biliary tree to the duodenal lumen. This target is obtained simply with biliary sphincterotomy. The choice of biliary stent placement has only few singular indications: 
I. The eventual cohesistence of a stenosis of the common bile duct (i.e. the surgical clips on the cystic stump are too close to the choledocal wall or a postoperative inflammation of the common bile duct)

\section{A perforation type II during the ERCP}

III. The association with acute/chronic pancreatitis

IV. The discovery at ERCP of a not previously diagnosed choledocolithiasiasis that the endoscopist cannot solve during the procedure (i.e. big stones impacted in the common bile duct)

V. The discovery at ERCP of a not previously diagnosed choledocal stenosis

In fact, in the case of common bile duct's stenosis, the biliary sphincterotomy cannot be enough effective because the pressure gradient may remain high: the stent would get better the inconvenience. If during ERCP, the endoscopistrealizes the presence of a perforation near the biliary orifice (i.e. outflow of the contrast medium), this is the case of well-known complication: the ERCP perforation type II and the stent is mandatory to treat it. The last two cases are referred to patients without an accurate preoperative imaging study with a missed diagnosis of choledocolithiasis or common bile duct stenosis: in these situations, a stent placement is suggested. In the case of common bile duct's stones, the endoscopic removal is crucial to remove the obstacle in the duct which increases the intrabiliary pressure and, so on, the pressure gradient. In case of multiple lithiasis or complicated ones (i.e. big stones not removable with usual devices neither by mechanical lithotripsy), a stent placement with or without a nose-biliary drain should be placed before a second-look procedure. At list, if a pancreatitis is present, the stent placement can be justified by the high risk of flogistic stenosis of the common bile duct associated to the inflammation of the gland. In the papers present in the literature, these indications are not discussed even mentioned and what emerges from our analysis is that many colleagues believe that the treatment of choice of cystic stump's bile leaks is the association of biliarysphincterotomy and stent placement. If it is not true, they have treated only patients with particular clinical conditions but they have forgotten to specify it in the articles.

Moreover, inserting a biliary stent if it is not necessary, can lead to a well-known condition: a recurrent bile leak from the cystic stump. The cause of the bile leak's persistency is, in many cases, the presence of the stent itself: it can increase the pressure gradient because the obstruction leads to an increase of the biliary pressure. In other cases, the presence of the surgical drain do not let the inflow of the bile in the duodenal lumen because the lesser pressure gradient is between the biliary pressure and the atmospheric one: pulling out or remove the drain will solve the inconvenience (Figure 1). It is a question of striking a balance!

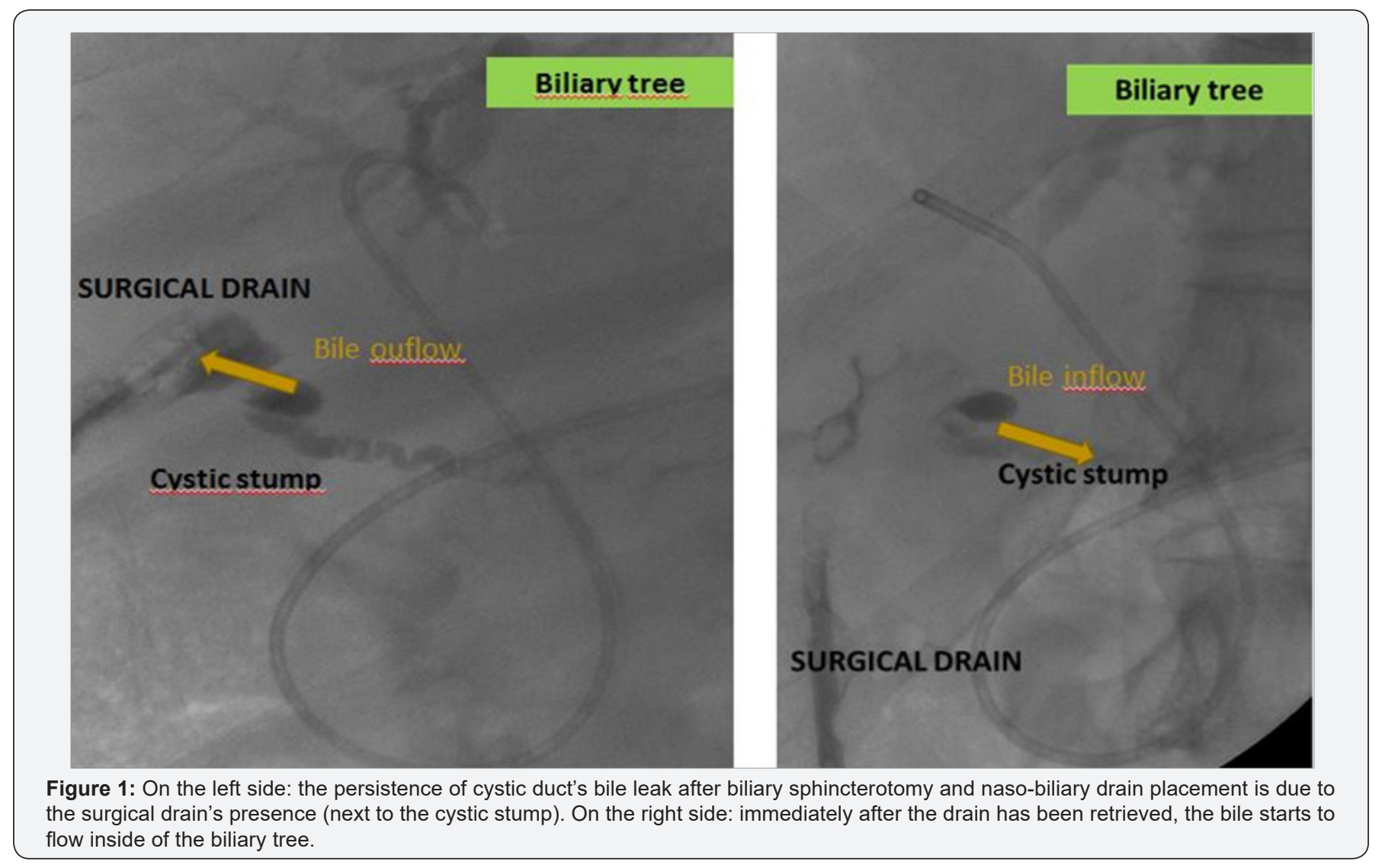




\section{References}

1. Yabe S, Kato H, Mizukawa S, Akimoto Y, Uchida D, Seki H, et al (2017) Predictive factors for outcomes of patients undergoing endoscopic therapy for bile leak after hepatobiliary surgery. Dig Endosc 29(3): 353-361.

2. Mutignani M, Shah SK, Tringali A, Perri V, Costamagna G (2002) Endoscopic therapy for biliary leaks from aberrant right hepatic ducts severed during cholecystectomy. GastrointestEndosc 55(7): 932-936.

3. Kook HK, Tae NK (2014) Endoscopic management of bile leakage after cholecystectomy: a single-center experience for 12 years. ClinEndosc 47(3): 248-253.

4. Rustagi T, Aslanian HR (2014) Endoscopic management of biliary leaks after laparoscopic cholecystectomy. J Clin Gastroent 48: 674-678.

5. Adler DG, Papachristou GI, Taylor LJ, McVay T, Birch M, et al. (2017) Clinical outcomes in patients with bile leaks treated via ERCP with regard to the timing of ERCP: a large multicenter study. Gastrintest Endosc 85(4): 766-772.

6. Martin D, Uldry E, Demartines N, Halkie N (2016) Bile duct injuries after laparoscopic cholecystectomy: 11-year experience in a tertiary center. Bio Science Trends 35(10): 197-201.

7. Ibrarullah M, Sankar S, Sreenivasan K, Gavini SRK (2015) Management of bile duct injury at various stages of presentation: experience from a tertiary care centre. Indian J Surg 77(2): 9298.
This work is licensed under Creative Commons Attribution 4.0 License DOI: $10.19080 /$ ARGH.2017.06.555678 